\title{
Vertical sleeve gastrectomy improves ventilatory drive through a leptin-dependent mechanism
}

\author{
Deanna M. Arble, ${ }^{1,2}$ Alan R. Schwartz, ${ }^{3}$ Vsevolod Y. Polotsky, ${ }^{3}$ Darleen A. Sandoval, ${ }^{2}$ \\ and Randy J. Seeley² \\ 'Department of Biological Sciences, Marquette University, Milwaukee, Wisconsin, USA. ${ }^{2}$ Department of Surgery, University \\ of Michigan, Ann Arbor, Michigan, USA. ${ }^{3}$ Division of Pulmonary and Critical Care and Sleep Medicine, Department of \\ Medicine, School of Medicine, Johns Hopkins University, Baltimore, Maryland, USA.
}

\begin{abstract}
Obesity hypoventilation syndrome (OHS) is a serious disorder characterized by daytime hypercapnia, disordered breathing, and a reduction in chemosensitivity. Vertical sleeve gastrectomy (VSC), a bariatric surgical procedure resulting in weight loss and weight-independent improvements in glucose metabolism, has been observed to substantially improve sleep-disordered breathing. However, it is unclear if the ventilatory effects of VSC are secondary to weight loss or the marked change in metabolic physiology. Using preclinical mouse models, we found that VSC leads to an improvement in the hypercapnic ventilatory response (HCVR) and reductions in circulating leptin levels independent of reductions in body mass, fat mass, and caloric intake. In the absence of leptin, VSC continues to improve body mass, fat mass, and glucose tolerance in ob/ob mice but no longer affects HCVR. However, the HCVR of $o b / o b$ mice can be returned to wild-type levels with leptin treatment. These data demonstrate that VSC improves chemosensitivity and ventilatory drive via a leptin-dependent mechanism. Clinically, these data downgrade the relative contribution of physical, mechanical load in the pathogenesis of OHS, and instead point to physiological components of obesity, including alterations in leptin signaling, as key drivers in OHS.
\end{abstract}

Conflict of interest: DAS receives funding from Ethicon Endo-Surgery, Novo Nordisk, and Boehringer Ingelheim International. R|S receives research support from Ethicon Endo-Surgery/ Johnson \& Johnson, Novo Nordisk, Janssen/Johnson \& Johnson, Zafgen, Medlmmune, and Kallyope.

License: Copyright 2019, American Society for Clinical Investigation.

Submitted: August 24, 2018 Accepted: November 29, 2018 Published: January 10, 2019

Reference information: JCI Insight. 2019;4(1):e124469. https://doi.org/10.1172/jci. insight.124469.

\section{Introduction}

Obesity hypoventilation syndrome (OHS) effects approximately $30 \%$ of the obese population and if untreated increases mortality by 2.5 -fold (1). A diagnosis of OHS requires the presentation of obesity (e.g., body mass index $[\mathrm{BMI}] \geq 30 \mathrm{~kg} / \mathrm{m}^{2}$ ), daytime elevations in the partial pressure of carbon dioxide $\left(\mathrm{PaCO}_{2}\right)$, reductions in oxygen $\left(\mathrm{PaO}_{2}\right)$, and sleep-disordered breathing. Of those diagnosed with $\mathrm{OHS}, 90 \%$ exhibit obstructive sleep apnea (OSA) (2). Individuals with OHS also exhibit reductions in their ventilatory response to hypercapnia and hypoxia, pointing to a centrally impaired chemosensitivity response (2).

Undoubtedly, obesity is critical in the pathogenesis of OHS. Clinical analysis of the relationship between $\mathrm{BMI}$ and $\mathrm{PaCO}_{2}$ in $\mathrm{OHS}$ individuals indicates that hypercapnia is unlikely to occur in an individual with a BMI below $30 \mathrm{~kg} / \mathrm{m}^{2}$ (2). Moreover, individuals with OHS who undergo weight loss exhibit modest improvements in OHS (3), including reductions in daytime $\mathrm{PaCO}_{2}$ and improved chemosensitivity (4). However, it is unclear if the mechanical load of obesity and/or the metabolic disturbances imposed by obesity contribute to the blunted chemosensitivity and disordered breathing observed in individuals with OHS.

Previous clinical research indicates that fat mass imposes a physical, mechanical load on respiratory muscles which in turn can lead to hypoventilation (5). However, increases in fat mass rarely occur without significant changes to an individual's metabolic physiology. Of note, obesity results in significantly elevated leptin levels and, in some cases, glucose intolerance. Leptin, a satiety hormone positively correlated to fat mass, conveys adiposity information to the brain. Interestingly, leptin has been found to compensate for increased mechanical loads on the diaphragm by stimulating the ventilatory drive in $o b / o b$ mice (6). It is possible that a breakdown in leptin signaling, potentially through obesity-induced leptin resistance (7), directly contributes to the pathogenesis of OHS independent of the physical, mechanical load of obesity. Indeed, a number of preclinical approaches have linked leptin action to chemosensitivity and disordered breathing (8-11). Alternatively, leptin may indirectly improve chemosensitivity and disordered breathing 
through its effect on glucose metabolism (12), as both clinical and preclinical studies have reported links between glycemic control and insulin action on chemosensitivity and disordered breathing (13).

Observations from patients undergoing bariatric surgery suggest that weight-independent changes in obese physiology contribute to improvements in disordered breathing such as OSA. Bariatric surgical procedures, such as the vertical sleeve gastrectomy (VSG) and the laparoscopic adjustable gastric band (LAGB) lead to significant, sustained weight loss and improvements in glucose regulation (14). However, VSG uniquely improves obesity-related physiology, including glucose metabolism and leptin levels, through both weight-dependent and -independent mechanisms (15). This contrasts with the LAGB, where metabolic improvements parallel total weight loss $(14,15)$. Interestingly, OSA resolution 1 year after VSG is approximately twice that of individuals undergoing LAGB $(16,17)$, suggesting that improvements in obesity-related physiology may improve OSA above and beyond the effects of physical body fat loss. Although few clinical studies have specifically examined the effects of bariatric surgery on OHS, they report that bariatric surgery leads to acute and long-term improvements in arterial blood gases $(18,19)$; however, it is unclear if these improvements are due to physical body weight loss or overall improvements in metabolic physiology. The role of leptin in chemosensitivity and disordered breathing following bariatric surgery is largely unknown.

In the present study, we tested the hypothesis that VSG affects chemosensitivity and ventilatory drive through a leptin-dependent mechanism using diet-induced obese wild-type (WT) mice, genetically obese, leptin-deficient $o b / o b$ mice, and our verified model of VSG (20). Using whole-body plethysmography, we determined baseline ventilation and the hypercapnia ventilatory response (HCVR) as preclinical markers of disordered breathing reflecting the alterations observed in OHS and OSA patients $(2,21-24)$. Within diet-induced obese WT mice, we found that VSG leads to an improvement in chemosensitivity and reductions in leptin, independent of body and fat mass. Leptin-deficient $o b / o b$ mice undergoing VSG fail to exhibit an improvement in HCVR; however, this can be restored with leptin treatment. Importantly, while $o b / o b$ mice undergoing VSG demonstrate significant reductions in body mass, fat mass, and improvements in glucose metabolism, leptin deficiency prevents VSG-induced improvements in chemosensitivity. We conclude that VSG improves chemosensitivity and ventilatory drive through a leptin-dependent mechanism.

\section{Results}

The effect of VSG on body mass, fat mass, and caloric intake in WT mice. Prior to surgical intervention, high-fat diet (HFD), pair-fed, and VSG groups were maintained on an HFD to induce obesity and all groups were assessed for ventilation and HCVR (Figure 1A). The HFD led to an approximately 16-g increase in body mass and an approximately 15-g increase in fat mass (Figure 1, B-D). On the day of surgery (day 0), mice underwent either VSG or sham surgery (Figure 1A). Additionally, on day 0 mice in the pair-fed group began caloric restriction to match the caloric intake of mice receiving the VSG (Figure 1A). Following surgery, mice received follow-up ventilatory measures and a glucose tolerance test (Figure 1A). Surgery acutely decreased body mass and fat mass in all animals maintained on the HFD (Figure 1, B-D). Three weeks after surgical intervention, pair-fed and VSG animals maintained a significant and similar reduction in body mass and fat mass (Figure 1, B-D) without altering lean mass (Supplemental Figure 1; supplemental material available online with this article; https://doi.org/10.1172/jci.insight.124469DS1). As designed, cumulative caloric intake at 4 days and 3 weeks after surgical intervention was similar between pair-fed and VSG mice (Figure 1E) to control for weight loss from the bariatric surgical procedure. VSG led to significant and sustained decreases in body mass and fat mass in part through a reduction in caloric intake. Importantly, pair-fed and VSG mice exhibited similar body mass and fat mass 4 days and 3 weeks after surgery. Therefore, any differences observed between pair-fed and VSG groups would be indicative of weight-independent effects of the VSG bariatric surgical procedure.

The effect of VSG on glucose regulation in WT mice. In response to an oral gavage of glucose performed 4 weeks after surgery, VSG and pair-fed mice exhibited an overall improvement in glucose tolerance in comparison with HFD-fed mice (Figure 1F). Blood glucose levels of HFD mice were significantly higher than all other groups 30 and 60 minutes following the glucose gavage (Figure 1F). Furthermore, HFD-fed mice also had elevated glucose values compared with chow and pair-fed groups prior to and 10 and 120 minutes following the glucose gavage. As expected, VSG mice exhibited a distinct glucose tolerance curve, including elevated glucose 15 minutes after gavage and a significant decrease at 30 minutes, which has been observed in other studies (25) (Figure 1F). Chow and pair-fed mice had similar glucose tolerance areas 
A
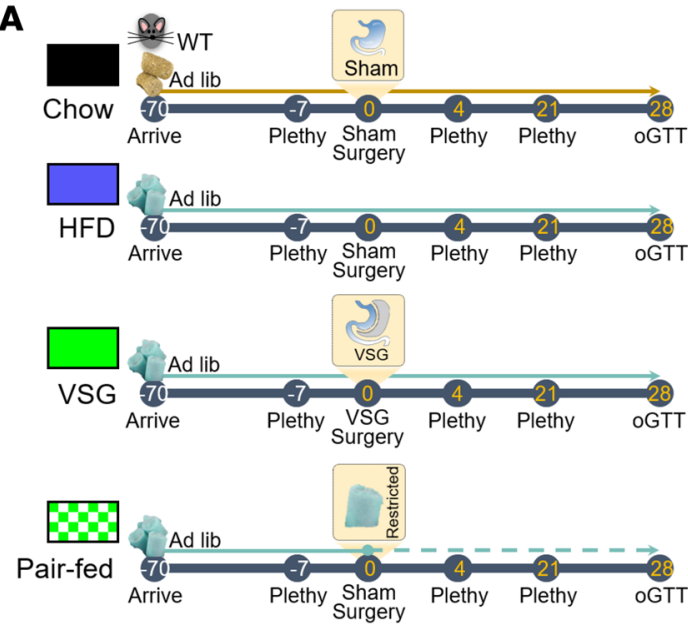

C
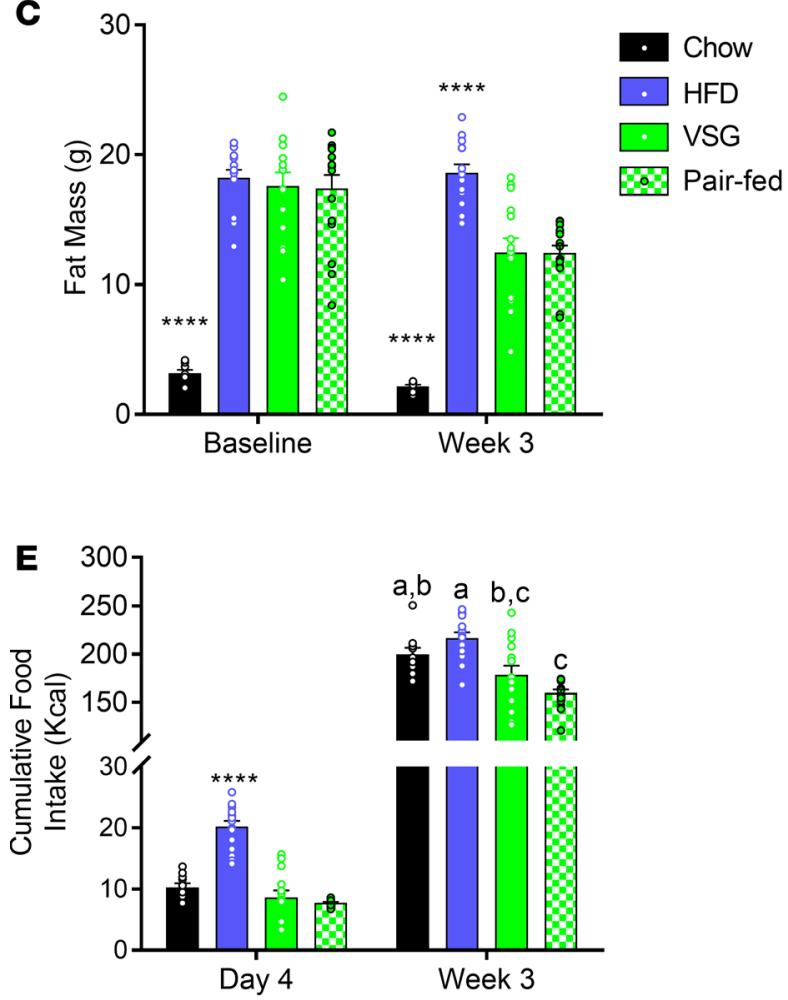

B
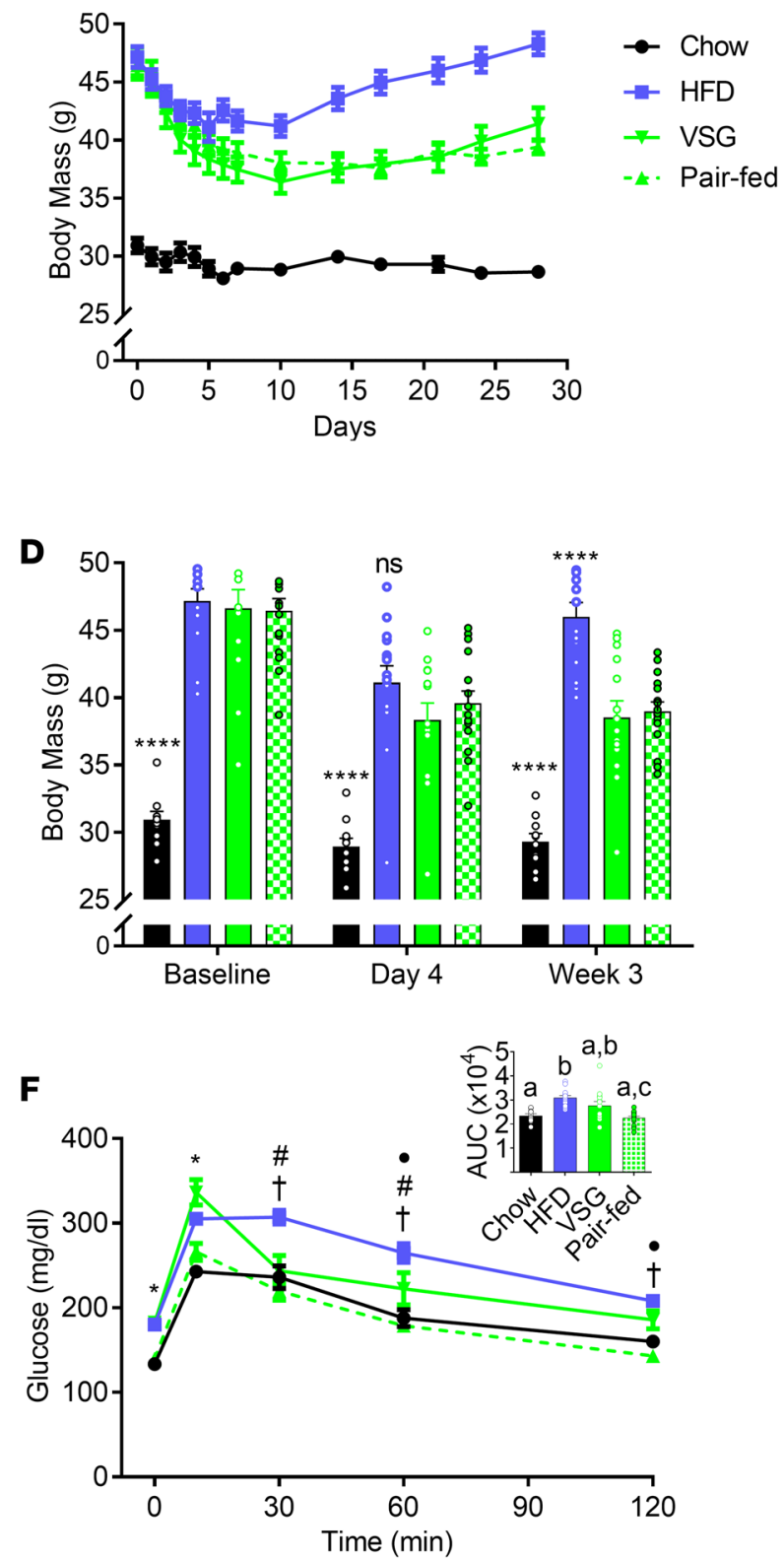

Figure 1. Body mass, fat mass, caloric intake, and glucose tolerance of WT mice following VSG. Generalized protocol and group description (A), body mass (B and $\mathbf{D})$, fat mass (C), cumulative caloric intake (E), glucose tolerance in response to a mixed-meal oral gavage (oGTT, F), and corresponding area under the curve (AUC, $\mathbf{F}$ insert) for WT, chow-fed mice receiving sham surgery (Chow, $n=10$, black lines/bars), high-fat diet-fed mice receiving sham surgery (HFD, $n=14-15$, blue lines/bars), high-fat diet-fed mice receiving vertical sleeve gastrectomy surgery (VSC, $n=14$, green lines/bars), and high-fat diet pair-fed mice receiving calories equivalent to the VSG group (Pair-fed, $n=16$, dotted green lines/bars). ${ }^{* * *} P<0.0001$ versus all other groups at the same time point. ${ }^{*} P<0.05$, Chow versus HFD, Chow versus VSG, HFD versus Pair-fed, Pair-fed versus VSG. $\dagger P<0.05$, HFD versus Chow and Pair-fed. ${ }^{*} P<0.05$, HFD versus VSG. ${ }^{\$} P<0.05$, Pair-fed versus VSG. Different letters indicate significant differences $(P<0.05)$ between groups. $P$ values indicate Tukey's post hoc comparisons from repeated-measures ANOVA or 1-way ANOVA. Data expressed as mean \pm SEM. Plethy, plethysmograph.

under the curve (AUCs), whereas VSG exhibited an intermediate AUC not significantly different from HFD or pair-fed/chow sham groups (Figure 1F). Thus, in this study, VSG led to improved glucose dynamics without a significant improvement in AUC.

The effect of VSG on ventilation and the HCVR in WT mice. Chemosensitivity in response to elevated carbon dioxide (i.e., hypercapnia) was assessed by ventilatory response (i.e., the HCVR) expressed as the slope of minute ventilation versus carbon dioxide concentration. Prior to surgical intervention (i.e., at baseline), ventilation 

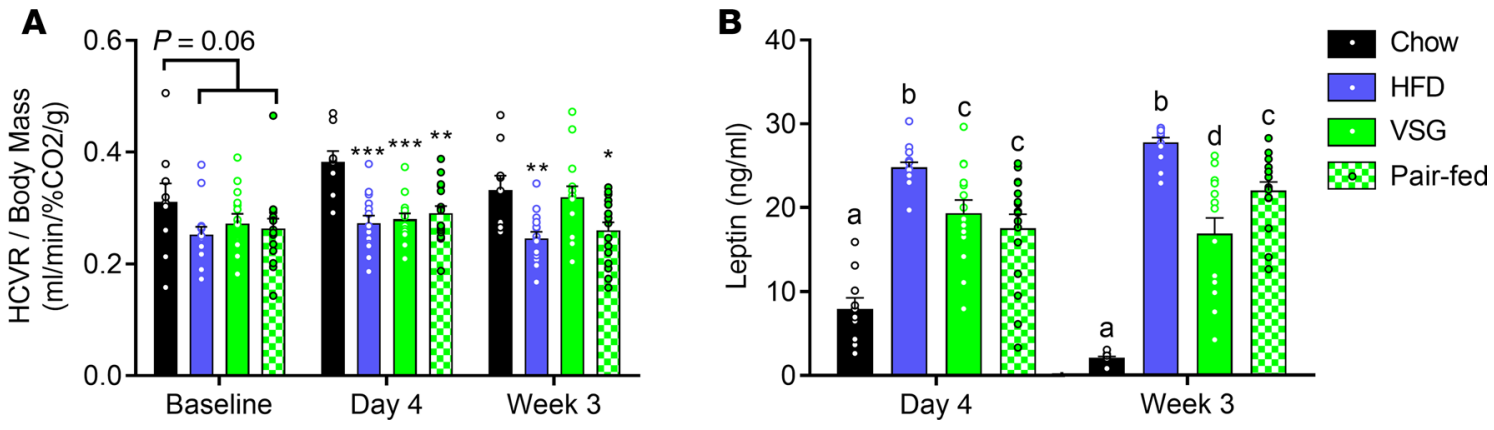

Figure 2. Hypercapnic ventilatory response and leptin levels of WT mice following VSG. Hypercapnic ventilatory response (HCVR) per gram body mass $\left(\mathrm{ml} / \mathrm{min} / \% \mathrm{CO}_{2} / \mathrm{g}\right)(\mathbf{A})$, and circulating leptin levels ( $\left.\mathrm{ng} / \mathrm{ml}\right)(\mathbf{B})$ in WT, chow-fed mice receiving sham surgery (Chow, $n=10$, black bars), high-fat diet-fed mice receiving sham surgery (HFD, $n=15$, blue bars), high-fat diet-fed mice receiving vertical sleeve gastrectomy surgery (VSG, $n=14$, green bars), and high-fat diet pair-fed mice receiving calories equivalent to the VSG group (Pair-fed, $n=16$, dotted green bars). ${ }^{*} P<0.05,{ }^{* *} P<0.01,{ }^{* * *} P<0.001$ versus Chow. Different letters indicate significant differences $(P<0.05)$ between groups. $P$ values indicate Tukey's post hoc comparisons from 1-way ANOVA. Data expressed as mean \pm SEM.

in response to a hypercapnic challenge was higher in diet-induced obese mice (Supplemental Figure 2D). This is consistent with clinical data that demonstrate that obesity increases HCVR to protect against the respiratory depression associated with an increased body mass $(26,27)$. Diet-induced obesity also reduced baseline ventilation frequency in normal room air conditions, without effecting minute ventilation or tidal volume (Supplemental Figure 2, A-C). After surgical intervention, the animals exhibited significantly different body masses over time in addition to different body masses between groups. Since HCVR is known to be affected by body mass (28), we normalized each animal's HCVR to its body mass at the time of testing (Figure 2A) to better determine how HCVR responds to our specific experimental manipulations. Clinically, a similar procedure is done by normalizing HCVR to an individual's surface area or height (29), allowing one to draw conclusions about chemosensitivity relative to increasing body mass. When expressed as HCVR per gram body mass, chemosensitivity compensation was similar between obese and lean mice prior to any surgical intervention (Figure 2A). However, we observed a trend $(P=0.06)$ for decreased chemosensitivity relative to body mass within obese mice, suggesting that obesity itself may lead to impairments in chemosensitivity compensation in alignment with OHS. This trend becomes significant 4 days after surgical intervention, when all animals maintained on the HFD (i.e., HFD, pair-fed, and VSG groups) exhibited a significant decrease in their ability to mount an HCVR appropriate for their body mass (Figure 2A). At this time, HFD, pair-fed, and VSG groups were of similar weight and weighed significantly more than the chow control group (Figure $1 \mathrm{~B}, P<0.0001$ ). Three weeks following surgical intervention, only animals undergoing the VSG were able to mount a chemosensitivity response appropriate for their body mass and similar to that of a lean control (Figure 2A). Importantly, at this time, HCVR was increased in animals receiving the VSG compared with those in the pair-fed group, despite both groups having a similar body mass, fat mass, and caloric intake (Figure 1, B-E). Taken together, these data demonstrate that chemosensitivity compensation per gram body mass is reduced with obesity (as observed within HFD and pair-fed groups) but can be corrected with VSG independent of changes in body mass and/or fat mass. However, weight loss alone is unable to improve HCVR or chemosensitivity compensation per gram body mass. This observation suggests that weight-independent component(s) of the VSG may restore chemosensitivity and the HCVR.

The effect of VSG on leptin in WT mice. Owing to leptin's involvement in ventilatory drive and HCVR $(11,13,30)$, we examined circulating plasma leptin levels directly following ventilatory testing 4 days and 3 weeks after surgical intervention. As expected, leptin was lowest in lean, WT mice and highest in HFD mice (Figure 2B). Pair-fed and VSG mice exhibited intermediate leptin levels, lower than HFD but higher than chow. Interestingly, 4 days after surgical intervention, pair-fed and VSG leptin levels were lower than HFD (Figure $2 \mathrm{~B}, P<0.05$ ), although all groups were of similar weight. Three weeks after surgical intervention, when pair-fed and VSG groups exhibited similar body mass and fat mass (Figure 1C), leptin was significantly lower in mice undergoing VSG (Figure $2 \mathrm{~B}, P<0.05$ ). Indeed, mice undergoing VSG had significantly less leptin $(\mathrm{ng} / \mathrm{ml})$ per gram fat mass $(1.31 \pm 0.06 \mathrm{~g})$ compared with pair-fed mice $(1.78 \pm 0.06 \mathrm{~g}, P<0.0001)$. These data point to the ability of the VSG surgery to alter leptin levels independent of body weight or fat mass in a manner consistent with decreased leptin resistance (7) 
The effect of VSG on body mass, fat mass, and caloric intake in leptin-deficient ob/ob mice. To determine if leptin was responsible for the WT's improvements in HCVR following VSG, we repeated the experimental design in leptin-deficient $o b / o b$ mice (Figure 3A). All ob/ob mice were maintained on a standard chow diet and received either sham or VSG surgery. Additionally, a leptin-treatment group received daily leptin (10 $\mu \mathrm{g} /$ day) and a pair-fed group was calorically restricted to match the caloric intake of leptin-treated mice. Prior to surgical intervention, all groups were of similar weight (average $47.5 \pm 0.6 \mathrm{~g}$, Figure $3 \mathrm{~A}$ ) and had similar fat and lean mass (Figure 3C and Supplemental Figure 3). As expected, sham control ob/ob mice gained weight over the course of the study; however, all other groups lost weight over the course of the study (Figure 3, B-D). Acutely, 5 days after surgical intervention, there were no significant differences in body mass between the groups (Figure 3, B and D). However, at 3 weeks after surgical intervention, there were significant differences in body mass and fat mass. Sham control mice weighed the most and had the highest fat mass (Figure 3, B-D; $P<0.01$ ). Leptin-treated mice weighed the least, with the corresponding least amount of fat mass (Figure 3, B-D; $P<0.05$ ). VSG and pair-fed groups exhibited equal body mass and fat mass, intermediate between sham control and leptin groups (Figure 3, B-D). There was also a small yet significant reduction in lean mass within the pair-fed group 3 weeks after surgical intervention (Supplemental Figure 3), perhaps due to an exaggerated catabolic response to the caloric restriction (31) in the absence of leptin signaling. Body mass and fat mass loss in leptin-treated and pair-fed mice is in part supported by a reduction in cumulative caloric intake (Figure 3E, $P<0.05$ ). Leptin-treated and pair-fed animals, by design, consumed similar calories over the course of the experiment. Sham control exhibited higher caloric intake by 5 days and 3 weeks after surgical intervention. While VSG showed a trend toward a reduction in cumulative caloric intake, it was not significantly different from sham control. These data demonstrate 2 key observations: (a) leptin reduces food intake and induces weight loss beyond caloric reduction alone, most likely via increases in energy expenditure (32); and (b) VSG induces weight loss independent of leptin. Importantly, because leptin, pair-fed, and VSG groups demonstrate significant weight loss, these data allow one to determine how glucose tolerance and chemosensitivity are specifically affected by leptin or VSG independent of other confounding variables such as body mass.

The effect of VSG on glucose regulation in ob/ob mice. In response to an oral gavage of glucose, sham control $o b / o b$ mice exhibited impaired glucose tolerance in comparison with all other groups (Figure 3F, $P<0.05)$. Strikingly, despite an approximately 10-g difference in body weight, VSG and leptin groups exhibited similar glucose tolerance, yet both were profoundly improved compared with sham control (Figure $3 \mathrm{~F}, P<0.05$ ). Moreover, pair-fed $o b / o b$ mice did not demonstrate an improvement in glucose tolerance, despite losing weight and weighing roughly the same as the VSG group (Figure 3F). Taken together, these data demonstrate that the VSG improves glucose tolerance in part through body mass-, fat mass-, and leptin-independent mechanisms.

The effect of VSG on ventilation, the HCVR, and leptin in ob/ob mice. Prior to surgical intervention, all ob/ob mice exhibited a similar HCVR (Supplemental Figure 4D), approximately half that of WT mice (Supplemental Figure 2D). Baseline ventilation under room air conditions was not significantly different between $o b / o b$ groups (Supplemental Figure 4, A-C). Five days after surgical intervention, and before a divergence of body weights between the groups, HCVR per gram body mass was exclusively increased within mice receiving leptin (Figure $4 \mathrm{~A}, P<0.05)$. Three weeks after surgical intervention, mice within the leptin group continued to be the only $o b / o b$ mice to experience an elevated chemosensitivity compensation per gram of body mass (Figure 4A, $P<$ 0.0001). Plasma leptin levels were verified 3 weeks after surgical intervention, immediately after plethysmography. As expected, all $o b / o b$ mice, except those treated with leptin, had leptin levels below the level of detection (Figure 4B). It is notable that leptin-treated $o b / o b$ mice and WT chow mice exhibited identical HCVRs $(9.6 \pm 0.3$ vs. $9.6 \pm 0.7 \mathrm{ml} / \mathrm{min} / \% \mathrm{CO}_{2}$; 2-tailed $t$ test, $\left.P=0.96\right) 3$ weeks following surgical intervention (Supplemental Figure $2 \mathrm{D}$ and Supplemental Figure $4 \mathrm{D})$, a time in which both groups exhibited similar body mass $(29.31 \pm 0.6 \mathrm{vs}$. $30.46 \pm 1.1 \mathrm{~g}$ ) and leptin levels $(2.07 \pm 0.2$ vs. $3.233 \pm 0.6 \mathrm{ng} / \mathrm{ml}$ ) (2-tailed $t$ tests, $P=0.38$ and $P=0.09$, respectively), although the leptin-treated $o b / o b$ mice had more fat mass $(2.16 \pm 0.1 \mathrm{vs.} 5.61 \pm 0.9 \mathrm{~g}$; 2-tailed $t$ test, $P<$ 0.01). Pair-fed $o b / o b$ mice exhibited a blunted HCVR over the course of the experiment and, like their WT counterparts, failed to increase chemosensitivity compensation per gram body mass (Figure 4A, $P>0.05$ ). Unlike WT animals, $o b / o b$ mice undergoing VSG did not experience an increase in chemosensitivity compensation per gram body mass and, indeed, exhibited a blunted HCVR throughout the experiment (Figure 4A). This observation is particularly intriguing given that there were no chronic, significant differences in body mass $(38.5 \pm 1.2$ vs. $40.1 \pm 2.1 \mathrm{~g} ; P=0.52$ ) or fat mass $(12.5 \pm 1.1$ vs. $15.7 \pm 1.6 \mathrm{~g} ; P=0.12)$ between WT VSG (Figure $1, \mathrm{~B}-\mathrm{D}$ ) 
A
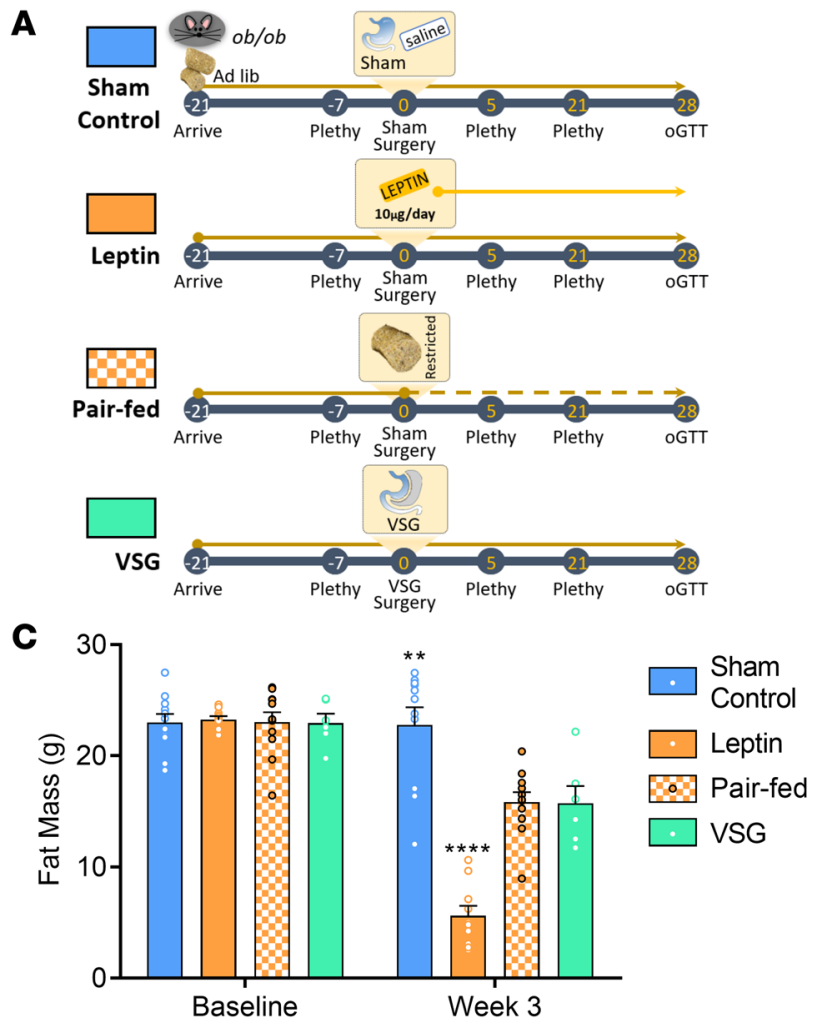

E

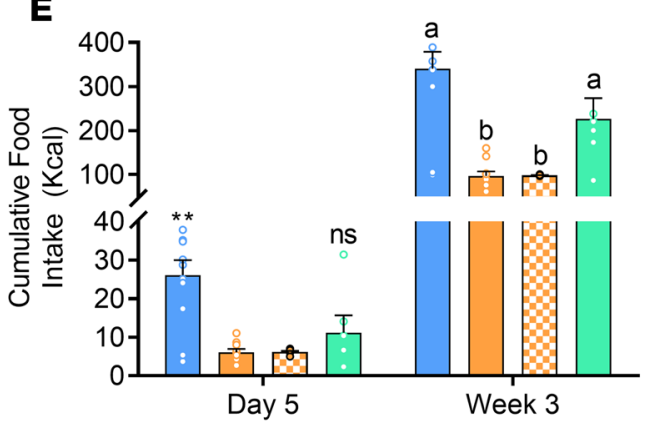

B

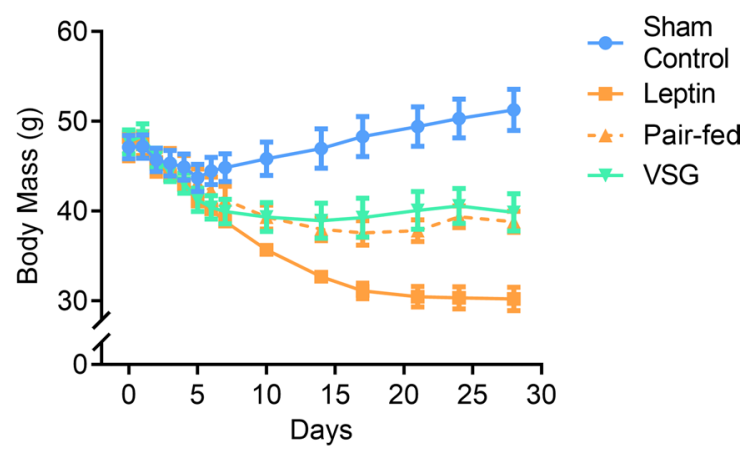

D
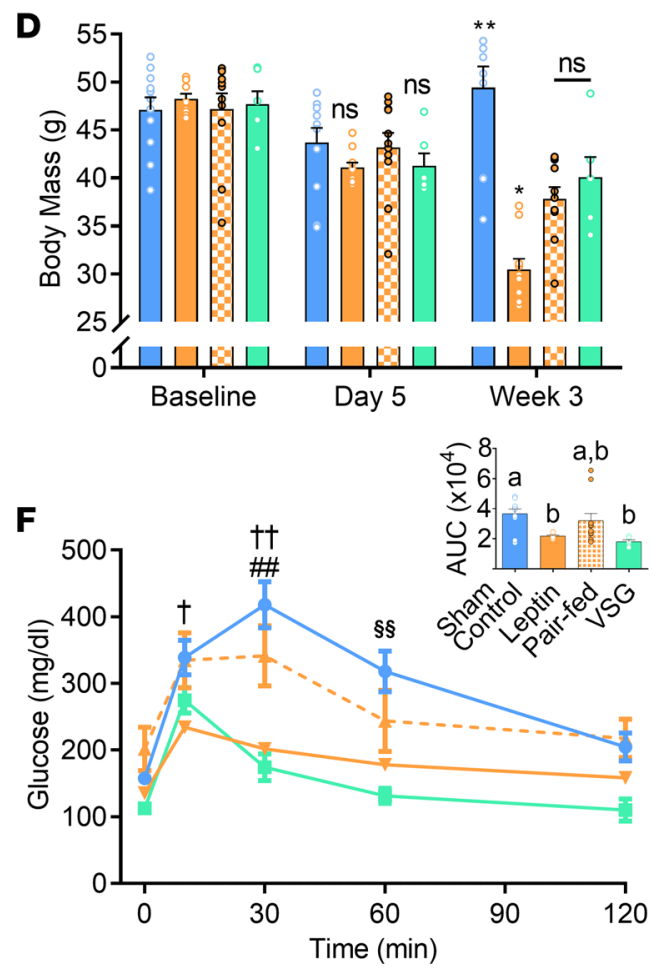

Figure 3. Body mass, fat mass, caloric intake, and glucose tolerance of leptin-deficient ob/ob mice following VSG. Generalized protocol and group description (A), body mass (B and $\mathbf{D})$, fat mass (C), cumulative caloric intake (E), glucose tolerance in response to a mixed-meal oral gavage (oGTT, F), and corresponding area under the curve (AUC, $\mathbf{F}$ insert) for chow-fed ob/ob mice receiving sham surgery (Sham Control, $n=11$, light blue lines/bars), daily leptin treatment (Leptin, $n=10$, orange lines/bars), pair-feeding yoked to leptin group (Pair-fed, $n=11$, dotted orange lines/bars), or vertical sleeve gastrectomy surgery (VSG, $n=6$, light green lines/bars). ${ }^{*} P<0.05,{ }^{* *} P<0.01$, ${ }^{* * *} P<0.0001$ versus all other groups at the same time point. $\dagger P<0.05, \dagger+P<0.01$ Leptin versus Sham Control and Pair-fed. ${ }^{\#} P<0.01$ VSG versus Sham Control and Pair-fed. ${ }^{\$} \$ P<0.01$ Sham Control versus Leptin and VSG. Different letters indicate significant differences $(P<0.05)$ between groups. $P$ values indicate Tukey's post hoc comparisons from repeated-measures ANOVA or 1-way ANOVA. Data expressed as mean \pm SEM. Plethy, plethysmograph.

and $o b / o b$ VSG (Figure 3, B-D) groups, underscoring the importance of leptin in the ventilatory response to hypercapnia independent of body and fat mass. Finally, since VSG leads to leptin-independent improvements in glucose metabolism without improving HCVR, it suggests that improvements in HCVR require leptin action and are not dependent on other confounding improvements in glucose metabolism.

These data highlight a number of key findings: (a) leptin treatment rescues ventilation and chemosensitivity of $o b / o b$ mice independent of body and fat mass; (b) as observed in WT mice, weight loss alone is unable to improve HCVR or chemosensitivity compensation per gram body mass; (c) VSG is unable to increase HCVR and chemosensitivity compensation without leptin signaling; and (d) improvements in glucose metabolism alone are insufficient to improve HCVR. 
A

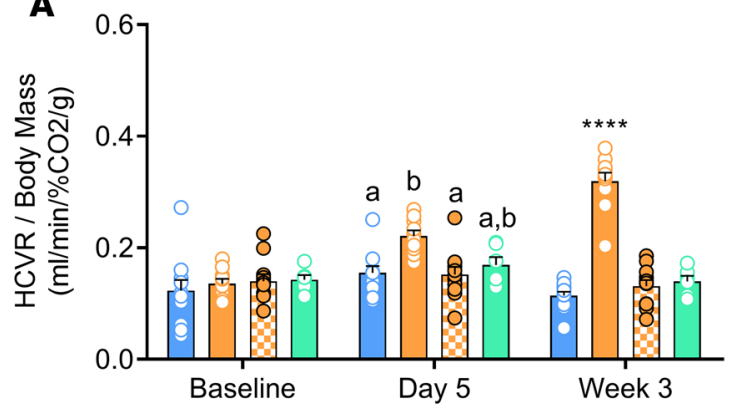

B

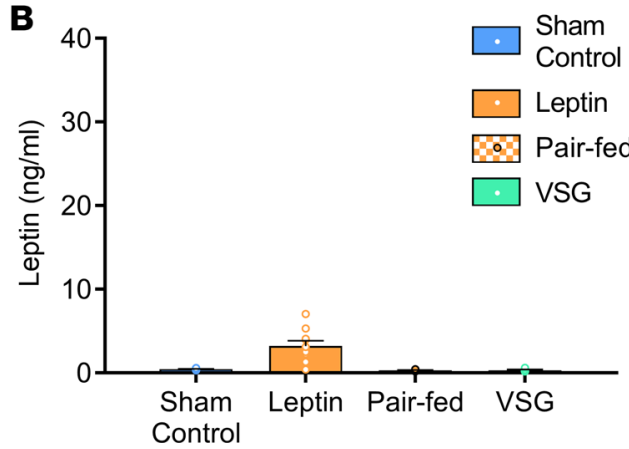

Figure 4. Hypercapnic ventilatory response and leptin levels of leptin-deficient ob/ob mice following VSG. Hypercapnic ventilatory response (HCVR) per gram body mass ( $\left.\mathrm{ml} / \mathrm{min} / \% \mathrm{CO}_{2} / \mathrm{g}\right)(\mathbf{A})$, and circulating leptin levels $(\mathrm{ng} / \mathrm{ml})(\mathbf{B})$ in chow-fed ob/ob mice receiving sham surgery (Sham Control, $n=11$, light blue bars), daily leptin treatment (Leptin, $n=10$, orange bars), pair-feeding yoked to leptin group (Pair-fed, $n=11$, dotted orange bars), or vertical sleeve gastrectomy surgery (VSG, $n=6$, light green bars). ${ }^{* * * *} P<0.0001$ versus all other groups at the same time point. Different letters indicate significant differences $(P<0.05)$ between groups. $P$ values indicate Tukey's post hoc comparisons from 1-way ANOVA. Data expressed as mean \pm SEM.

\section{Discussion}

In the current study, we segregate the mechanical load of obesity from the metabolic disturbances imposed by obesity by utilizing diet-induced obese mice undergoing VSG or weight-matched caloric restriction. As a metabolic surgery, the VSG procedure is known to modulate obese physiology (including glycemic control and leptin levels) independent from weight loss. Thus, this model offers a unique opportunity to explore the physical versus the physiological aspects of obesity and the specific effects on disordered breathing and chemosensitivity. In WT mice, we found that VSG leads to improvements in disordered breathing and chemosensitivity appropriate for the animals' body mass that are not observed in weight-matched controls. To our knowledge, this is the first study to report on ventilation and/or chemosensitivity following metabolic surgery in a preclinical mouse model. Yet, our data are in agreement with clinical observations that individuals undergoing bariatric or metabolic surgery exhibit a profound improvement in disordered breathing shortly after surgery (33) and improvements in OHS symptoms $(18,19)$. We further show that VSG is unable to improve ventilation or chemosensitivity in leptin-deficient $o b / o b$ mice. In agreement with previous reports $(11,30)$, exogenous leptin treatment in $o b / o b$ mice restores a WT ventilation response before significant body/fat mass is lost. Together, these results indicate that VSG can improve ventilatory control and chemosensitivity independent of body mass and through a leptin-dependent mechanism.

Strikingly, we did not observe an improvement in ventilatory drive from weight-loss alone. While the traditional clinical assumption has been that nonsurgical weight loss would lead to improvements in OHS on par with surgically induced weight loss (5), to our knowledge these data have not been specifically reported. Instead, most of the observations regarding the effect of weight loss on OHS has been from morbidly obese, bariatric surgery patients. Although it remains to be tested within the clinical population, the present study would suggest that nonsurgical weight loss (e.g., caloric restriction) would not have the same effect on OHS symptoms as metabolic surgery, even if the same amount of weight loss could be achieved by each method. Thus, these data shed light on the contribution of mechanical load in the pathogenesis of OHS, downgrading the relative importance of physical weight and underscoring the physiological contribution in the pathogenesis of OHS.

While the mechanism by which metabolic surgery leads to weight-independent changes in physiology remain an active research question, a predominant view is that metabolic surgery exerts its actions via the brain. This hypothesis is validated by a number of studies showing that metabolic surgery can alter complex behaviors, such as addiction (34) and food choice (35), as well as dramatic changes in neurohormonal gut peptides (36) including an almost 10-fold increase in glucagon-like peptide 1 (GLP-1) (37). However, not all physiological changes following VSG are critical for weight loss or glucose regulation. For example, despite the known involvement of GLP-1 in glucose regulation, mouse models lacking GLP-1 receptors specifically within $\beta$ cells (37) or throughout the whole body (38) successfully lose weight and exhibit an improvement in glucose tolerance following VSG. Similarly, while leptin is not necessary for the weight loss or metabolic outcomes following VSG $(39,40)$, leptin is altered following metabolic surgery (41). In agreement with these observations, we report VSG-induced improvements in glucose tolerance in $o b / o b$ mice and reductions in WT leptin levels, both effected independent of weight loss. 
Notably, while metabolic surgery does not result in an increased metabolic response to leptin administration (e.g., a decrease in food intake) (41), it remains possible that leptin's action on nonmetabolic parameters such as disordered breathing and chemosensitivity is improved following VSG. We have recently demonstrated that modulating leptin signaling at the neuronal level, specifically within the periaqueductal gray, leads to a disordered-breathing phenotype and a reduction in HCVR (42). This opens the possibility that VSG not only affects the brain's control of feeding behaviors and glucose regulation, but ventilation and chemosensitivity as well. As mounting evidence suggests that metabolic surgeries can improve glucose regulation through weight-independent pathways, it is hardly surprising that other pathophysiological states associated with obesity may also be improved.

It is possible that our interpretation of these data is skewed by the disproportional loss of $o b / o b$ mice as compared to WT mice following VSG. The excluded animals may have exhibited differential responses and the surviving mice thus biased the results. While we cannot be certain of the former, we can report that the excluded $o b / o b$ mice did not demonstrate any initial weight, food intake, or other observable differences from those that completed the study. In regard to the latter, the number of $o b / o b$ mice that completed the study was sufficient to draw statistical comparisons and our metabolic conclusions are in agreement with others' reports $(39,40)$.

These data provide evidence that the pathogenesis of OHS is rooted in physiological mechanisms that, like metabolic surgery (14), go beyond physically restrictive mechanisms and implicate alterations in the gut-brain axis. The ability of a peripheral surgery to affect complex physiology and behavior has profound implications for future therapeutic targets to treat obesity, OHS, and other disordered breathing such as OSA. Indeed, these results open the possibility for metabolic surgeries and potentially leptin to be used as novel treatments for chemosensitivity and disordered breathing.

\section{Methods}

Study design. To determine the effect of body weight and VSG on disordered breathing and leptin levels, we utilized C57BL6/J WT male mice divided into 4 groups: (a) A chow sham group was maintained on a standard chow diet and received a sham surgery to mirror nonspecific effects of the VSG surgery. (b) An HFD sham group was maintained on a $45 \% \mathrm{kcal}$ HFD before and after receiving a sham surgery. (c) An HFD VSG group was maintained on a $45 \% \mathrm{kcal}$ diet before and after receiving the VSG surgery. (d) An HFD pair-fed group was pair-fed with the VSG group, meaning that mice in HFD pair-fed group received the same amount of kcal per day as mice in the HFD VSG group elected to eat. In this way, the HFD pairfed group was on a restricted calorie diet matching the calories experienced by the VSG mice and could control for VSG-independent weight loss.

To determine if VSG can lead to leptin-independent effects on disordered breathing, leptin-deficient $o b / o b$ mice were divided into 4 groups. Owing to the $o b / o b$ mouse's genetic propensity toward obesity, an HFD is not necessary to induce obesity; all $o b / o b$ mice were maintained on a standard chow diet. Group 1: A sham group was implanted with a mini-osmotic pump delivering vehicle (water) and received a sham surgery to mirror nonspecific effects of the VSG surgery. Group 2: A VSG group was implanted with a mini-osmotic pump delivering vehicle (water) and received the VSG surgery. Group 3: A leptin group was implanted with a mini-osmotic pump delivering leptin and received a sham surgery. Group 4: A pair-fed group was pair-fed with the leptin group, meaning that mice in the pair-fed group received the same amount of kcal per day as mice in the leptin group elected to eat. In this way, the pair-fed group was on a restricted calorie diet matching the weight loss and allowed one to make conclusions about weight loss independent of VSG.

For the outlined experiments, sample size was determined based on previous validated mouse studies using the VSG model (43). For all non-VSG groups, the final analyzed $n$ ranged from $10-16$ per group. For VSG groups, the final analyzed $n$ was $14 \mathrm{C} 57 \mathrm{BL} 6 / \mathrm{J}$ mice and $6 o b / o b$ mice. Among all the C57BL6/J mice, 7 out of $62(11 \%)$ were excluded from analysis due to failure to recover from the VSG surgery $(n=3)$ or from intestinal obstruction and/or infection $(n=4)$. Among all ob/ob mice, 12 out of $50(24 \%)$ were excluded from analysis due to failure to recover from the VSG surgery $(n=8)$, from intestinal obstruction and/or infection $(n=3)$, or death from an unknown cause $(n=1)$. The relatively high number of $o b / o b$ mice failing to recover from the VSG is believed to be independent of the VSG procedure itself. Instead, we believe the recovery rate in $o b / o b$ mice is affected by the mouse's genetic propensity to hypothermia. $O b / o b$ mice have been observed to have lower body temperatures and energy expenditure (44). 
For all collected measurements, except body weight and daily food intake measures, the experimenter was blinded to the experimental group. For measurement of body weight and food intake, it is necessary to know the group information as an additional health check for recovery after the surgery.

Animals. Mice were housed in a standard vivarium room under a standard 12-hour light/12-hour dark cycle. C57BL6/J (WT) and $o b / o b$ mice were ordered from the Jackson Laboratory at 6-8 weeks of age. All mice were singly housed. To induce obesity in diet-induced obese WT mice, mice were fed an HFD (45\% fat, $4.54 \mathrm{kcal} / \mathrm{g}$, D12451 Research Diets) for 10 weeks before surgery and were maintained on the same diet after surgery. All mice had ad libitum access to food, with the exception of the pair-fed groups that were maintained on a calorically restricted diet. For pair-fed mice, the restricted food amount was calculated daily based on the average intake of the paired group (i.e., WT VSG or $o b / o b$ Leptin) and administered once per day. For all studies, groups were age matched and divided to ensure equal body weights among similarly genotyped animals prior to surgical intervention.

Baseline ventilation and HCVR. To measure baseline respiration and the response to hypercapnia in freely moving, conscious mice we used a whole-body plethysmograph (Emka Technologies). A mouse was placed inside the plethysmograph chamber and habituated to its new environment for 30 minutes prior to measurement collection (only one mouse was placed inside a single chamber at a given time). During the habituation period, air exchange allows the animal to breath room air. Next, animals were exposed to a mixture of $21 \%$ oxygen and 79\% nitrogen (to mirror room air conditions) and baseline respiration, including breathing rate, inspiration/expiration time, volume and flow (relative changes from baseline), relaxation time, end-inspiration and end-expiration pause, and enhanced pause were recorded. Then, the chamber gases were changed to deliver hypercapnic environments over 4-minute increments (similar to the protocol used in ref. 11) at 3\%, $5 \%$, and $8 \%$ carbon dioxide separated by 5 -minute recovery periods of a room air mix. For all hypercapnic exposures, oxygen was maintained at $21 \%$ and nitrogen concentration was modified to allow for changes in carbon dioxide. Similar respiratory measures were recorded as during baseline ventilation. Animals were monitored continuously and kept awake by gentle tapping on the chamber to prevent sleep-associated changes in HCVR (11). HCVR was calculated by the slope of $\mathrm{CO}_{2} \%$ versus minute ventilation.

Glucose tolerance test. For the oral glucose tolerance test, all animals were fasted for 6 hours prior to an oral gavage of $200 \mu 1$ Ensure Plus (Abbott Laboratories). All glucose was measured by glucometer prior to gavage/injection (time 0), and then 15, 30, 60, and 120 minutes after gavage/injection. Blood was collected from the tip of the tail by cutting a small amount of the tail and gently massaging the blood out. Animals were excluded from analysis based on pre-established criteria, i.e., if blood glucose did not rise significantly over the 2-hour period or if the presence of diarrhea was observed, indicating a failed glucose gavage. One mouse was excluded from the test due to the presence of diarrhea (a WT HFD mouse).

VSG. All mice were exposed to liquid Osmolite (Abbott Laboratories) before surgery. Surgery began when mice were 11-16 weeks old and followed surgical procedures as previously indicated (45). Briefly, after isoflurane anesthesia, all animals (including those in the sham surgery condition) received a midline abdominal skin incision, followed by an incision in the underlying muscle wall. VSG mice had approximately $80 \%$ of the stomach resected along the major curvature, while sham mice had their stomach exposed and manipulated but not cut. Following surgery, mice were maintained on liquid Osmolite for 4 days, solid food was returned on the fourth day along with Osmolite, and on the fifth day mice were only provided solid food from then on. Metacam was provided daily for 4 days after surgery for pain.

Leptin treatment. Leptin was delivered by mini-osmotic pump (Allzet) for 6 weeks at a rate of 10 $\mu \mathrm{g} /$ day. This dose of leptin was selected in an effort to achieve circulating leptin levels similar to WT chow-fed control mice. Pumps were implanted subcutaneously in the scapular region of the mouse during the time of VSG or sham surgery. Vehicle pumps, delivering water, were implanted into all $o b / o b$ mice not receiving leptin treatment. In accordance with the manufacturer's guidelines, pumps reached steady-state release in approximately 48 hours. Therefore, 5 days after surgery is equivalent to day 3 of steady-rate leptin treatment.

Body mass, fat mass, and food intake measurements. Body weight and food intake measurements were measured over the course of the experiment daily or 2 times per week. Food was measured by manually removing food from the cage to determine weight. Total fat and lean mass were calculated using a whole-body composition analyzer (EchoMRI).

Leptin measurements. Blood was drawn for leptin measurements immediately after exposure to the hypercapnia challenge in the whole-body plethysmograph. Plasma was collected and tested via a Mouse 
Leptin Kit (Meso Scale Diagnostics). Blood was collected after hypocapnia exposure to limit stress prior to ventilation measurements.

Statistics. Statistical analysis was performed using GraphPad Prism version 5.0. Statistical significance was determined either by repeated measures or 1-way ANOVA followed by Tukey's post hoc test. Results were considered statistically significant when $P<0.05$ (2-tailed design).

Study approval. All studies were reviewed, approved by, and performed according to the guidelines of the Institutional Animal Care and Use Committee of the University of Michigan.

\section{Author contributions}

DMA designed and performed experiments, analyzed data, and wrote the manuscript. ARS, VYP, DAS, and RJS contributed to experimental design and edited the manuscript.

\section{Acknowledgments}

We thank A. Lewis, A. Myronovych, M. Toure, and D. Farris for technical assistance in performing the vertical sleeves and C. Misko for assistance in collection of ventilation data. This work was supported by the NIH, National Institute of Diabetes and Digestive and Kidney Diseases (DK082480-01, to DAS; and R01 DK093848-01, to RJS). VYP and ARS receive support from the National Heart, Lung, and Blood Institute (HL128970 to VYP and ARS; HL138932 and HL133100 to VYP).

Address correspondence to: Deanna Arble, Marquette University, Wehr Life Sciences Building, Room 222, 530 N. 15th Street, Milwaukee, Wisconsin 532233 USA. Phone: 414. 288.7518; Email: deanna.arble@ marquette.edu.

1. Nowbar S, et al. Obesity-associated hypoventilation in hospitalized patients: prevalence, effects, and outcome. Am JMed 2004;116(1):1-7.

2. Mokhlesi B. Obesity hypoventilation syndrome: a state-of-the-art review. Respir Care. 2010;55(10):1347-1362.

3. Olson AL, Zwillich C. The obesity hypoventilation syndrome. Am J Med. 2005;118(9):948-956.

4. Rochester DF, Enson Y. Current concepts in the pathogenesis of the obesity-hypoventilation syndrome. Mechanical and circulatory factors. Am J Med. 1974;57(3):402-420.

5. Parameswaran K, Todd DC, Soth M. Altered respiratory physiology in obesity. Can Respir J. 2006;13(4):203-210.

6. Tankersley CG, et al. Leptin attenuates respiratory complications associated with the obese phenotype. J Appl Physiol. 1998;85(6):2261-2269.

7. Myers MG, Cowley MA, Münzberg H. Mechanisms of leptin action and leptin resistance. Annu Rev Physiol. 2008;70:537-556.

8. Manzella D, et al. Soluble leptin receptor and insulin resistance as determinant of sleep apnea. Int J Obes Relat Metab Disord. 2002;26(3):370-375.

9. Ip MS, Lam KS, Ho C, Tsang KW, Lam W. Serum leptin and vascular risk factors in obstructive sleep apnea. Chest. 2000;118(3):580-586.

10. Polotsky M, et al. Effects of leptin and obesity on the upper airway function. J Appl Physiol. 2012;112(10):1637-1643.

11. O'donnell CP, et al. Leptin prevents respiratory depression in obesity. Am J Respir Crit Care Med. 1999;159(5 Pt 1):1477-1484

12. Morton GJ, Schwartz MW. Leptin and the central nervous system control of glucose metabolism. Physiol Rev. 2011;91(2):389-411.

13. Framnes SN, Arble DM. The bidirectional relationship between obstructive sleep apnea and metabolic disease. Front Endocrinol (Lausanne). 2018;9:440.

14. Sandoval D. Bariatric surgeries: beyond restriction and malabsorption. Int J Obes (Lond). 2011;35 Suppl 3:S45-S49.

15. Arble DM, Sandoval DA, Seeley RJ. Mechanisms underlying weight loss and metabolic improvements in rodent models of bariatric surgery. Diabetologia. 2015;58(2):211-220.

16. Hutter MM, et al. First report from the American College of Surgeons Bariatric Surgery Center Network: laparoscopic sleeve gastrectomy has morbidity and effectiveness positioned between the band and the bypass. Ann Surg. 2011;254(3):410-420

17. Carlin AM, et al. The comparative effectiveness of sleeve gastrectomy, gastric bypass, and adjustable gastric banding procedures for the treatment of morbid obesity. Ann Surg. 2013;257(5):791-797.

18. Sugerman HJ, Baron PL, Fairman RP, Evans CR, Vetrovec GW. Hemodynamic dysfunction in obesity hypoventilation syndrome and the effects of treatment with surgically induced weight loss. Ann Surg. 1988;207(5):604-613.

19. Sugerman HJ, Fairman RP, Sood RK, Engle K, Wolfe L, Kellum JM. Long-term effects of gastric surgery for treating respiratory insufficiency of obesity. Am J Clin Nutr. 1992;55(2 Suppl):597S-601S.

20. Chambers AP, et al. Weight-independent changes in blood glucose homeostasis after gastric bypass or vertical sleeve gastrectomy in rats. Gastroenterology. 2011;141(3):950-958.

21. Javaheri S, Colangelo G, Lacey W, Gartside PS. Chronic hypercapnia in obstructive sleep apnea-hypopnea syndrome. Sleep. 1994;17(5):416-423.

22. Piper AJ, Grunstein RR. Big breathing: the complex interaction of obesity, hypoventilation, weight loss, and respiratory function. J Appl Physiol. 2010;108(1):199-205

23. Davis EM, O'Donnell CP. Rodent models of sleep apnea. Respir Physiol Neurobiol. 2013;188(3):355-361. 
24. Polotsky VY, et al. The impact of insulin-dependent diabetes on ventilatory control in the mouse. Am J Respir Crit Care Med. 2001;163(3 Pt 1):624-632.

25. Arble DM, Sandoval DA, Turek FW, Woods SC, Seeley RJ. Metabolic effects of bariatric surgery in mouse models of circadian disruption. Int J Obes (Lond). 2015;39(8):1310-1318.

26. Piper AJ, Grunstein RR. Obesity hypoventilation syndrome: mechanisms and management. Am J Respir Crit Care Med. 2011;183(3):292-298.

27. Chapman KR, Himal HS, Rebuck AS. Ventilatory responses to hypercapnia and hypoxia in patients with eucapnic morbid obesity before and after weight loss. Clin Sci. 1990;78(6):541-545.

28. Marcus CL, Glomb WB, Basinski DJ, Davidson SL, Keens TG. Developmental pattern of hypercapnic and hypoxic ventilatory responses from childhood to adulthood. J Appl Physiol. 1994;76(1):314-320.

29. Aitken ML, Franklin JL, Pierson DJ, Schoene RB. Influence of body size and gender on control of ventilation. J Appl Physiol. 1986;60(6):1894-1899.

30. O’Donnell CP, Tankersley CG, Polotsky VP, Schwartz AR, Smith PL. Leptin, obesity, and respiratory function. Respir Physiol. 2000;119(2-3):163-170

31. Aydin C, Jarema KA, Phillips PM, Gordon CJ. Caloric restriction in lean and obese strains of laboratory rat: effects on body composition, metabolism, growth and overall health. Exp Physiol. 2015;100(11):1280-1297.

32. Hwa JJ, et al. Leptin increases energy expenditure and selectively promotes fat metabolism in ob/ob mice. Am J Physiol. 1997;272(4 Pt 2):R1204-R1209.

33. Buchwald H, et al. Bariatric surgery: a systematic review and meta-analysis. JAMA. 2004;292(14):1724-1737.

34. Pepino MY, Stein RI, Eagon JC, Klein S. Bariatric surgery-induced weight loss causes remission of food addiction in extreme obesity. Obesity (Silver Spring). 2014;22(8):1792-1798.

35. Wilson-Pérez HE, et al. The effect of vertical sleeve gastrectomy on food choice in rats. Int J Obes (Lond). 2013;37(2):288-295.

36. Ochner CN, Gibson C, Shanik M, Goel V, Geliebter A. Changes in neurohormonal gut peptides following bariatric surgery. Int J Obes (Lond). 2011;35(2):153-166.

37. Douros JD, et al. Enhanced glucose control following vertical sleeve gastrectomy does not require a $\beta$-cell glucagon-like peptide 1 receptor. Diabetes. 2018;67(8):1504-1511.

38. Wilson-Pérez HE, et al. Vertical sleeve gastrectomy is effective in two genetic mouse models of glucagon-like peptide 1 receptor deficiency. Diabetes. 2013;62(7):2380-2385

39. Bachmann R, Lange J, Königsrainer A, Küper MA. Effects of sleeve gastrectomy in leptin deficient (ob/ob) mice and diet induced obese mice. Integrative Obesity and Diabetes website. https://www.oatext.com/effects-of-sleeve-gastrectomy-in-leptindeficient-ob-ob-mice-and-diet-induced-obese-mice.php\#Article. Accessed December 6, 2018.

40. $\mathrm{Li} \mathrm{F}$, et al. Preventative sleeve gastrectomy contributes to maintaining $\beta$ cell function in $\mathrm{db} / \mathrm{db}$ diabetic mouse. Obes Surg. 2016;26(10):2402-2410

41. Stefater MA, et al. Sleeve gastrectomy induces loss of weight and fat mass in obese rats, but does not affect leptin sensitivity Gastroenterology. 2010;138(7):2426-2436.

42. Flak JN, et al. A leptin-regulated circuit controls glucose mobilization during noxious stimuli. J Clin Invest. 2017;127(8):3103-3113

43. Chambers AP, et al. Similar effects of roux-en-Y gastric bypass and vertical sleeve gastrectomy on glucose regulation in rats. Physiol Behav. 2011;105(1):120-123.

44. Pelleymounter MA, et al. Effects of the obese gene product on body weight regulation in ob/ob mice. Science. 1995;269(5223):540-543.

45. Ryan KK, et al. FXR is a molecular target for the effects of vertical sleeve gastrectomy. Nature. 2014;509(7499):183-188. 